\title{
'LA CRÓNICA': UN MODELO QUE ARTICULA UNA PRENSA DE PROXIMIDAD GRATUITA EN ARAGÓN
}

\author{
Fernando Sabés Turmo \\ (Universitat Autònoma de Barcelona) \\ fernando.sabes@uab.es
}

\begin{abstract}
Resumen: "La Crónica" es una publicación comarcal y local que existe en Aragón desde hace 11 años y que se ha consolidado como un modelo de comunicación de proximidad en esta Comunidad Autónoma en la que tradicionalmente han sido muy escasos este tipo de proyectos. Esta publicación, que en realidad son 26 cabeceras distribuidas por las tres provincias aragonesas, busca abrirse un hueco con una apuesta por temas sociales, aunque la presencia de contenidos institucionales continúa siendo muy elevada. El análisis de este proyecto y sus perspectivas de futuro centrarán este artículo.
\end{abstract}

Palabras Clave: Prensa gratuita, "La Crónica”, comunicación local, Aragón.

Abstract: "La Crónica", is a local periodical in Aragón that for 11 years now has been consolidated as a model of proximity information in this region. In Aragon this type of proximity journalism has scarcely happened before. "La Crónica", which is made up of 26 mastheads distributed along the three provinces, tries to find a place in the market by paying attention to the social issues, though still institutional contents cover many pages. This RA sets out to analyse this periodical and its immediate future.

Keywords: Free Press, "La Crónica", local information, Aragón.

\section{INTRODUCCIÓN}

a prensa en papel está buscando su nuevo sitio en el mercado. Estas publicaciones deben innovar sus estrategias para conseguir ser rentables y poder mantener su actividad. La situación de crisis económica no hace sino acentuar esta necesidad de redefinir buena parte de sus líneas de actuación para ocupar una cuota de mercado y también publicitaria que les permita continuar existiendo.

Si la consolidación de la Sociedad de la Información está llevando a una redefinición de los productos impresos poniendo en cuestión cada vez más el soporte papel que está siendo sustituido a pasos agigantados por la red, la actual situación económica no hace sino condicionar todavía más el futuro de esas publicaciones periódicas en papel, ya sean diarias o no. De todos modos, hay que reconocer que el hueco para los productos próximos impresos todavía existe:

Internet ha provocado grandes debates en las redacciones de muchos periódicos. Entre los puntos a discusión se encuentran si las ediciones en línea deben tener primacía sobre 

gratuita en Aragón

las ediciones en papel o viceversa; si ambas deben tener o no redacciones separadas; si las ediciones electrónicas son una vía para ganar futuros compradores de papel o, por el contrario, si estimulan la fuga de probables consumidores. Cada medio ha tomado rumbos distintos. Usualmente ninguno ha durado. El debate sigue abierto y no hay respuestas definitivas a estos dilemas. $O$, dicho de otra manera, nadie tiene la receta sobre qué hacer. Otro factor que afecta la rentabilidad de los periódicos tradicionales es la proliferación de la prensa gratuita. Aunque usualmente estas publicaciones no son más que publicidad comercial disfrazada de información, crean la ilusión entre quienes lo leen de saber lo que pasa, sin tener que pagar por ella. Una ilusión, alimentada por la radio y la televisión (Hernández Navarro, 2009).

Esta delicada situación por la que actualmente pasa la prensa se acentúa más en territorios como Aragón, con una baja densidad de población y con unas características sociodemográficas que ponen en duda la viabilidad de algunos proyectos. Además, en esta Comunidad Autónoma, el escaso y peculiar tejido mediático también condiciona, y mucho, la viabilidad de algunas propuestas existentes y conduce a dudar de la puesta en marcha de nuevos proyectos que no tengan a las instituciones públicas como valedoras para garantizar su existencia.

En este complicado panorama ha surgido en los últimos años un nuevo modelo de prensa mayoritariamente mensual, comarcal y gratuita bajo la denominación de "La Crónica" y que es una decidida apuesta por la comunicación de proximidad, rompiendo con la estructura mediática tradicional existente en la Comunidad Autónoma y buscando un nuevo nicho de mercado que prácticamente no estaba explotado en la demarcación.

El objetivo de este trabajo es analizar esta nueva propuesta que se podría denominar de prensa autonómica, aunque segmentada con cabeceras en buena parte de las comarcas aragonesas y que también tiene en algunos barrios de la ciudad de Zaragoza.

\section{EL PANORAMA DE LOS MEDIOS LOCALES EN ARAGÓN: LA PRENSA}

La estructura de los medios de comunicación de proximidad en Aragón es muy débil. Tradicionalmente las grandes cabeceras de prensa diaria han sido las que han copado un sector donde en el ámbito más cercano las propuestas siempre han sido escasas y en buena parte de los casos publicaciones dependientes de los ayuntamientos, es decir, revistas municipales.

En el sector de la prensa diaria de pago, Heraldo de Aragón es la publicación con mayor impacto, seguida de El Periódico de Aragón, aunque hay que destacar también los casos de las provincias de Huesca y Teruel, donde sus diarios propios, Diario del Altoaragón y Diario de Teruel, tienen una extraordinaria aceptación. Ambos con propuestas de cercanía y con mucha información que se podría denominar de comarcas, lo que les permite una implantación territorial que en muchos casos es muy superior incluso a la de Heraldo y Periódico. 
Por su parte, los diarios gratuitos se han consolidado en las grandes ciudades aragonesas aunque no son ajenos a los problemas de supervivencia que sufre este tipo de prensa ante la reducción de los ingresos publicitarios debido esencialmente a la crisis económica.

Esta delicada situación de la comunicación de proximidad se puede trasladar también a los dos medios tradicionales audiovisuales: la radio y la televisión. Hasta el surgimiento de la Radio y Televisión Autonómica en 2005, eran escasas las estaciones de cercanía existentes.

En el caso de la radio, no se han localizado en los últimos años prácticamente operadores legales que no estén adscritos a alguna gran cadena, mientras que la dificultad de la implantación de la televisión local ha sido una tónica en la Comunidad Autónoma, debido a la falta de un mercado que realmente la pudiera hacer rentable económicamente y fuera de Zaragoza y Huesca capital, la viabilidad del resto de proyectos ha sido en todo momento más que complicada.

\section{APROXIMACIÓN AL FENÓMENO DE 'LA CRÓNICA'}

\subsection{Historia y organización empresarial}

El nacimiento de "La Crónica", como ejemplo de prensa comarcal gratuita en Aragón, se produjo en dos fases. La primera de ellas, en diciembre de 1998, cuando 134 Comunicación ${ }^{1}$ puso en marcha la edición en Barbastro y en la Comarca Somontano (provincia de Huesca) de "Vivir en Barbastro". Fue una de las primeras publicaciones comarcales gratuitas de Aragón. La segunda, sólo varios meses después, la protagonizó el Grupo Z en Aragón que impulsó "La Crónica".

Los orígenes de ambos proyectos son distintos. El primero, en la provincia de Huesca, parte de una empresa de comunicación con un perfil de agencia de publicidad y con una orientación hacia el cliente/anunciante aunque con estructura periodística para los contenidos. Por su parte, el proyecto del Grupo Z surgió con un mayor peso de los contenidos dada su propia estructura en la Comunidad Autónoma y como una forma también de proyectar sus productos periodísticos en todo el territorio aragonés.

Otra de las diferencias de ambas propuestas reside en su concepción territorial. En el caso de Huesca, la sede de 134 Comunicación se localiza en Barbastro, municipio de 18.000 habitantes, por lo que se articula un proyecto que se podría denominar desde abajo cubriendo de forma espontánea una necesidad en comu-

\footnotetext{
1134 Comunicación es una empresa de comunicación que nació pensada esencialmente en convertirse en una agencia de publicidad y que posteriormente ha centrado buena parte de su negocio en impulsar medios de comunicación de proximidad, esencialmente publicaciones gratuitas, aunque también ha creado emisoras de radio.
} 

gratuita en Aragón

nicación de lugares menos urbanos en los que no existía esa estructura y que generalmente adolecían de medios de comunicación impresos de referencia, a excepción de los diarios de pago y alguna cabecera privada que en su mayoría tenía un respaldo institucional directo. Por su parte, el proyecto del Grupo Z se dirigía desde Zaragoza, siguiendo un criterio distinto en cuanto a la cercanía e implicación en el territorio.

Ambas empresas llegaron a un acuerdo de intercambio en 2003, utilizando desde ese momento la cabecera de "La Crónica" para las diferentes publicaciones que se han ido consolidando desde ese momento en todo Aragón. Pese a ese acuerdo de colaboración, se mantiene la independencia de ambos respecto a la estructura económica o periodística, lo que ha llevado, incluso, a tomar decisiones diferentes entre ambos en determinados momentos. De esta forma, 134 Comunicación pasaba a gestionar las cabeceras de "La Crónica" en la provincia de Huesca, mientras que el Grupo Z, las de Zaragoza y Teruel.

Ese acuerdo empresarial llevó a una unificación en el diseño interno de las publicaciones, aunque se puedan encontrar pequeñas diferencias. La impresión se desarrollaba en la planta de impresión que el Grupo Z tenía en Zaragoza, pero desde el cierre de la misma, en 2009, todas las cabeceras se imprimen en la planta que Heraldo de Aragón tiene en la capital aragonesa.

Sí se desarrollan de forma común algunas estrategias comerciales que permiten aprovechar las sinergias entre ambos grupos. Por su parte, a nivel informativo, son dos estructuras diferentes, aunque con nexos de colaboración.

\subsection{La implantación en el territorio}

El punto probablemente más fuerte del proyecto de "La Crónica" es su implantación territorial. En buena parte de las 33 comarcas aragonesas se ha ubicado una cabecera pero también se ha dado el fenómeno de que en algún caso no ha cuajado y su existencia no ha sido posible, como es el caso de la Comarca de la Hoya, cuya capital es Huesca.

En total, son 30 las cabeceras que existen en el territorio aragonés con periodicidad mensual o menor, distribuyéndose siempre de forma gratuita. En total, la tirada mensual de esta prensa gratuita aragonesa ronda los 375.000 ejemplares (incluyendo los que se reparten una vez al mes y los que se hacen en diferentes ocasiones cada 30 días) que se distribuyen esencialmente mediante reparto individualizado en buzones y en depósito en lugares públicos.

\subsection{1. 'La Crónica' en la provincia de Huesca}

El fenómeno de "La Crónica" en la provincia de Huesca es gestionado, como ya se ha indicado, por el Grupo 134 Comunicación desde su sede en la localidad de Barbastro. Desde allí, y con una red de corresponsales, elabora siete cabeceras: Barbastro y el Somontano, Monzón y el Cinca Medio, La Litera, Bajo Cinca, Sobrarbe, Ribagorza, Alto Gállego y Monegros. 
Tabla 1. Cabeceras, tirada y periodicidad de 'La Crónica' en Huesca.

\begin{tabular}{|l|c|c|}
\hline Cabeceras & Tirada & Periodicidad \\
\hline Barbastro y el Somontano & 10.200 & Mensual \\
\hline Monzón y el Cinca Medio & 10.000 & Mensual \\
\hline Bajo Cinca & 9.000 & Mensual \\
\hline Alto Gállego & 7.500 & Mensual \\
\hline Monegros & 10.000 & Mensual \\
\hline La Litera & 7.500 & Mensual \\
\hline Ribagorza & 7.000 & Mensual \\
\hline Sobrarbe & 3.000 & Menual \\
\hline
\end{tabular}

Fuente: Elaboración propia.

Figura 1. Portada de 'La Crónica' de Barbastro y el Somontano

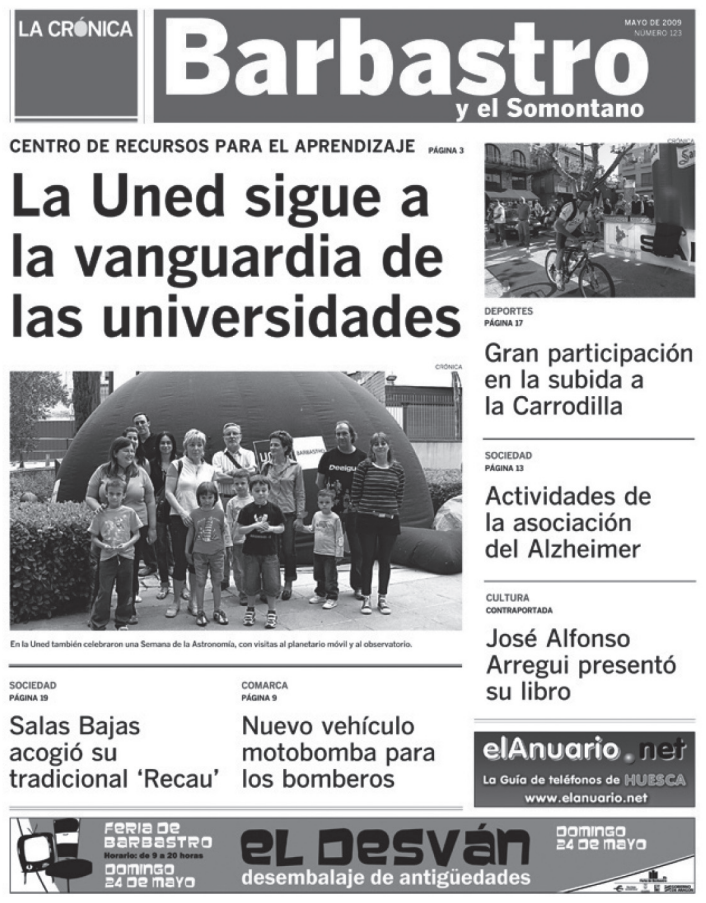


"La crónica": un modelo que articula una prensa de proximidad gratuita en Aragón

\subsection{2. 'La Crónica' en la provincia de Zaragoza}

La implantación de "La Crónica" en Zaragoza debe entenderse en dos ámbitos territoriales muy diferentes: Zaragoza ciudad y Zaragoza provincia. Seis son las cabeceras distintas de "La Crónica" que se reparten en la ciudad de Zaragoza. El Grupo Z pretende, de esta forma, captar un público y también unos anunciantes con un producto diferente dirigido a los habitantes de zonas concretas de la ciudad en los que combina información cercana con publicidad próxima, aunque en este caso también encontramos otra que denominaríamos genérica y que podría aparecer en cualquiera de las cabeceras analizada. La concentración poblacional de Aragón en su capital permite que se consolide un proyecto como éste pensando en la segmentación de públicos de Zaragoza.

Las cabeceras en la ciudad de Zaragoza son las siguientes: Actur, Rey Fernando y El Rabal; Distrito Rural Norte (Juslibol, Movera, Peñaflor, San Gregorio, San Juan de Mozarrifar, Santa Isabel y Montañana); Distrituo Rural Oeste (Alfocea, Casetas, Garrapinillos, Villarrapa, Miralbueno, Venta del Olivar, Monzalbarba y Utebo); Casco Histórico; Distrito Centro y Distrito Universidad.

Tabla 2. Cabeceras, tirada y periodicidad de 'La Crónica' en Zaragoza capital.

\begin{tabular}{|l|c|c|}
\hline Cabeceras & Tirada & Periodicidad \\
\hline Actur, Rey Fernando y El Rabal & 38.000 & 3 al mes \\
\hline Distrito Rural Norte & 8.500 & Mensual \\
\hline Distrito Rural Oeste & 12.000 & Mensual \\
\hline Casco Histórico & 15.000 & Mensual \\
\hline Distrito Centro & 16.000 & Mensual \\
\hline Distrito Universidad & 15.000 & \\
\hline
\end{tabular}

Fuente: Elaboración propia. 
Figura 2. Portadas de 'La Crónica' de Casco Histórico y Distrito Universidad, ambas de la ciudad de Zaragoza.
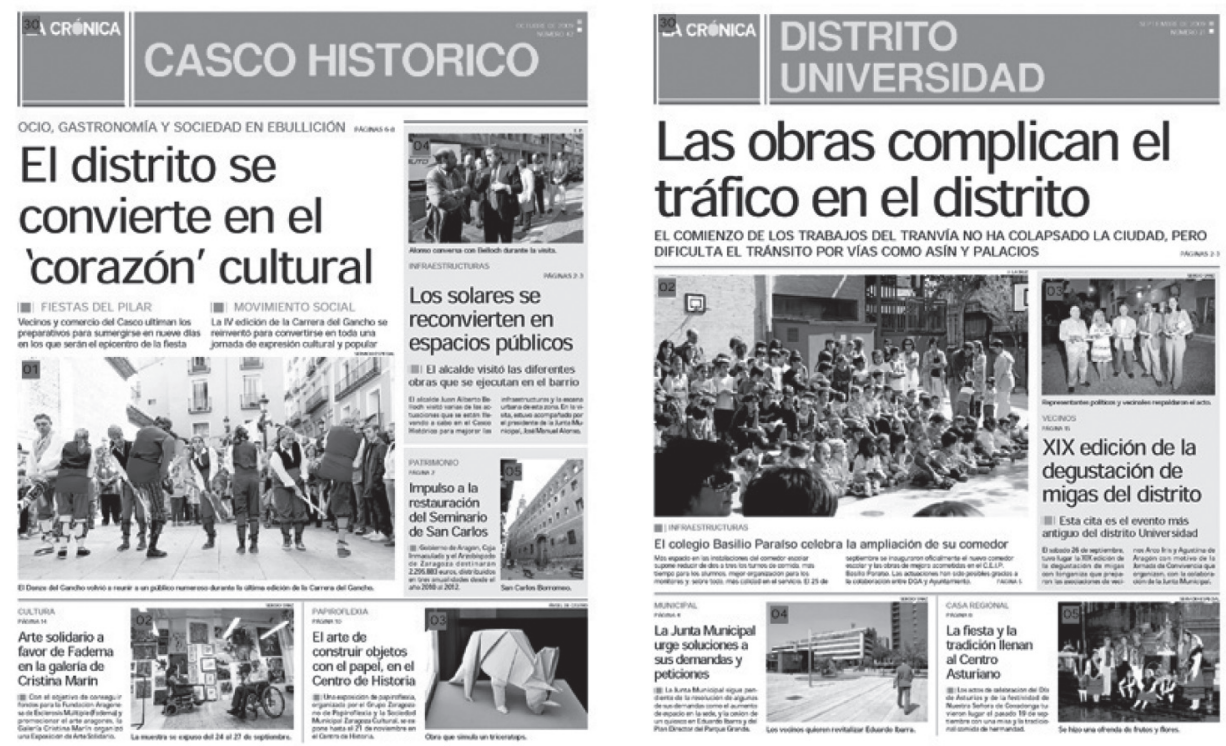

En cuanto a las publicaciones de la provincia de Zaragoza, hay que indicar que son 12 las cabeceras que aparecen y que vienen a cubrir un hueco informativo al que prácticamente el resto de medios impresos de la Comunidad Autónoma no hacen referencia. La mayor parte de las informaciones que recogen los diarios generalistas, e incluso también las publicaciones no diarias, se ciñen a contenidos que se generan en Zaragoza capital, centro neurálgico de la Comunidad Autónoma. Por este motivo, el papel de "La Crónica" adquiere mayor relevancia en cuanto a generador de información y de opinión en el marco de la comunicación de cercanía con el lector, al hacer especial hincapié en contenidos noticiables de proximidad y como un elemento estructurante de la comunicación local en Aragón, a través de publicaciones impresas.

Las 12 cabeceras de "La Crónica" en la provincia de Zaragoza, excluyendo la capital, son las siguientes: Valdejalón, Bajo Gállego, Campo de Borja, Ribera Izquierda del Ebro, Ribera Alta del Ebro, Comunidad de Calatayud, Tarazona y El Moncayo, Campo de Belchite, Ribera Baja del Ebro, Campo de Cariñena, Cinco Villas y Campo de Daroca. 

gratuita en Aragón

Tabla 3. Cabeceras, tirada y periodicidad de 'La Crónica' en Zaragoza provincia (excluida la capital).

\begin{tabular}{|l|c|c|}
\hline Cabeceras & Tirada & Periodicidad \\
\hline Valdejalón & 11.500 & Mensual \\
\hline Bajo Gállego & 6.000 & Mensual \\
\hline Campo de Borja & 8.800 & Bimestral \\
\hline Ribera Izquierda del Ebro & 4.000 & Mensual \\
\hline Ribera Alta del Ebro & 10.000 & Mensual \\
\hline Comunidad de Calatayud & 16.000 & Mensual \\
\hline Tarazona y el Moncayo & 6.200 & Bimestral \\
\hline Campo de Belchite & 2.500 & Mensual \\
\hline Ribera Baja del Ebro & 8.500 & \\
\hline
\end{tabular}

Fuente: Elaboración propia.

Figura 3. Portada de 'La Crónica' de Ribera Baja del Ebro.

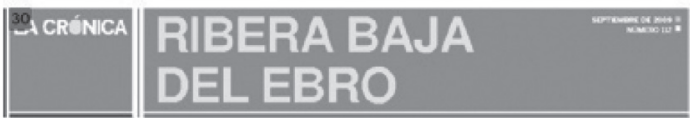

\section{La comarca potencia los servicios sociales \\ EL TRANSPORTE ADAPTADO FAVORECE LA CREACIÓN DE EMPLEO LOCAL}

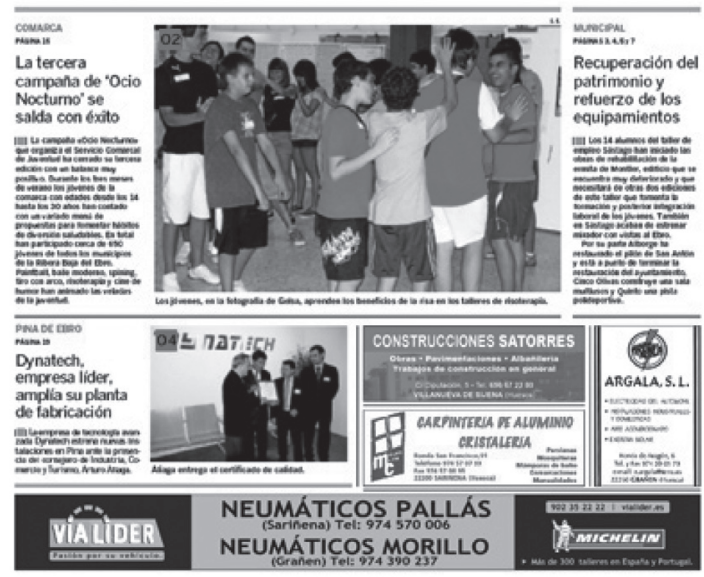




\subsection{3. 'La Crónica' en la provincia de Teruel}

"La Crónica" cuenta únicamente con tres cabeceras en la provincia de Teruel, probablemente el territorio aragonés con menor implantación de esta publicación. La mayor dispersión poblacional hace todavía más complicado la consolidación de un proyecto como éste de publicaciones gratuitas en un territorio con una baja tasa poblacional. De hecho, se pueden leer en Teruel las cabeceras de Cuencas Mineras, Bajo Aragón y Andorra Sierra de Arcos.

Tabla 4. Cabeceras, tirada y periodicidad de 'La Crónica' en Teruel.

\begin{tabular}{|l|c|c|}
\hline Cabeceras & Tirada & Periodicidad \\
\hline Cuencas Mineras & 4.500 & Mensual \\
\hline Bajo Aragón & 11.000 & Mensual \\
\hline Andorra Sierra de Arcos & 5.000 & Mensual \\
\hline
\end{tabular}

Fuente: Elaboración propia.

Figura 4. Portada de 'La Crónica' de la Comarca de las Cuencas Mineras.

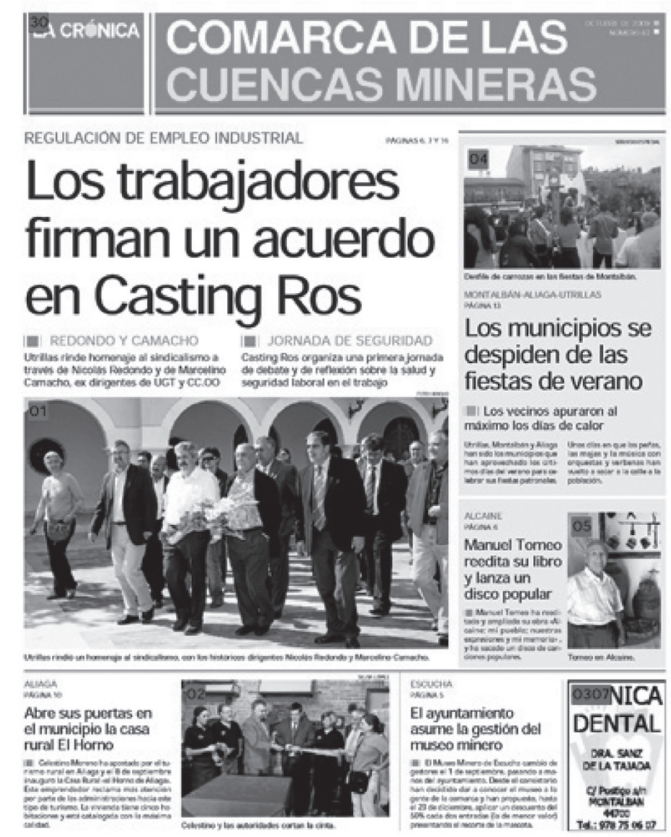


"La crónica": un modelo que articula una prensa de proximidad gratuita en Aragón

\subsection{Evolución del formato}

El formato de "La Crónica" siguió en su origen el diseño de El Periódico de Aragón, publicación diaria de pago del Grupo Z en la Comunidad Autónoma. Pasado un tiempo se evidenció la necesidad de mejorar el formato y se planteó un cambio de diseño que llegó entre febrero y marzo de 2009. Los motivos que llevaron a esta modificación fueron esencialmente dos: la adaptación a un modelo de formato ya habitual en la prensa gratuita, más pequeño que la tradicional y más visual, y una reducción de costos, dado que el cambio de formato implica un ahorro también en el consumo de papel.

El nuevo formato ha desarrollado innovaciones en el concepto gráfico del producto, con un menor contenido literario y un aumento de los contenidos gráficos. Se trata de una evolución lógica de acuerdo a los planteamientos de la prensa gratuita diaria y no diaria, así como a las propias exigencias del lector de este tipo de productos que demanda un planteamiento más visual de la cabecera. Hay que tener en cuenta que el lector tradicional de prensa tiene un perfil distinto y en las zonas donde se distribuye "La Crónica" no es mayoritaria ese tipo de audiencia.

Con los contenidos más visuales se busca dar cabida a las numerosas colaboraciones de lo que se podría denominar periodismo social que llegan a la redacción a través de distintos colectivos y asociaciones de los municipios de cada una de las zonas. El nuevo formato gráfico de los contenidos permite una mayor participación.

Xosé López (2000) en su artículo "El modelo local de la prensa gallega redefine estrategias para competir en la nueva era" reconocía el esfuerzo de las empresas periodísticas por mejorar sus productos, aunque ligaba este hecho al de la preocupación por los contenidos próximos. "Estas mejoras han estado acompañadas por una estrategia de atención a la información local como vía para reforzar la definición del producto y la identidad propia".

En la actualidad, el formato de una página de "La Crónica" es de 256×314 milímetros, a seis columnas y con 60 módulos de publicidad en cada una de ellas. El número mínimo de páginas de cada una de las cabeceras es 16 y crece de ocho en ocho páginas, por exigencias de la rotativa en la que se imprime. En el caso de que se vaya al número mínimo de páginas, 16, todas ellas son a color, mientras que si se aumenta dependerá de las exigencias de la publicidad.

En cuanto a las familias tipográficas utilizadas, hay que indicar que para los titulares se usa la Zimmer, mientras que para el texto, que aparece en un tamaño de 9,5 puntos, la SwiftND.

No existe una división estándar del producto por secciones. Pese a que pueden existir algunas comunes, como es el caso de deportes, los contenidos son los que marcan la existencia o no de estas divisiones. 
Figura 5. Portadas de 'La Crónica de la Litera' con el formato antiguo (izquierda) y el nuevo (derecha).
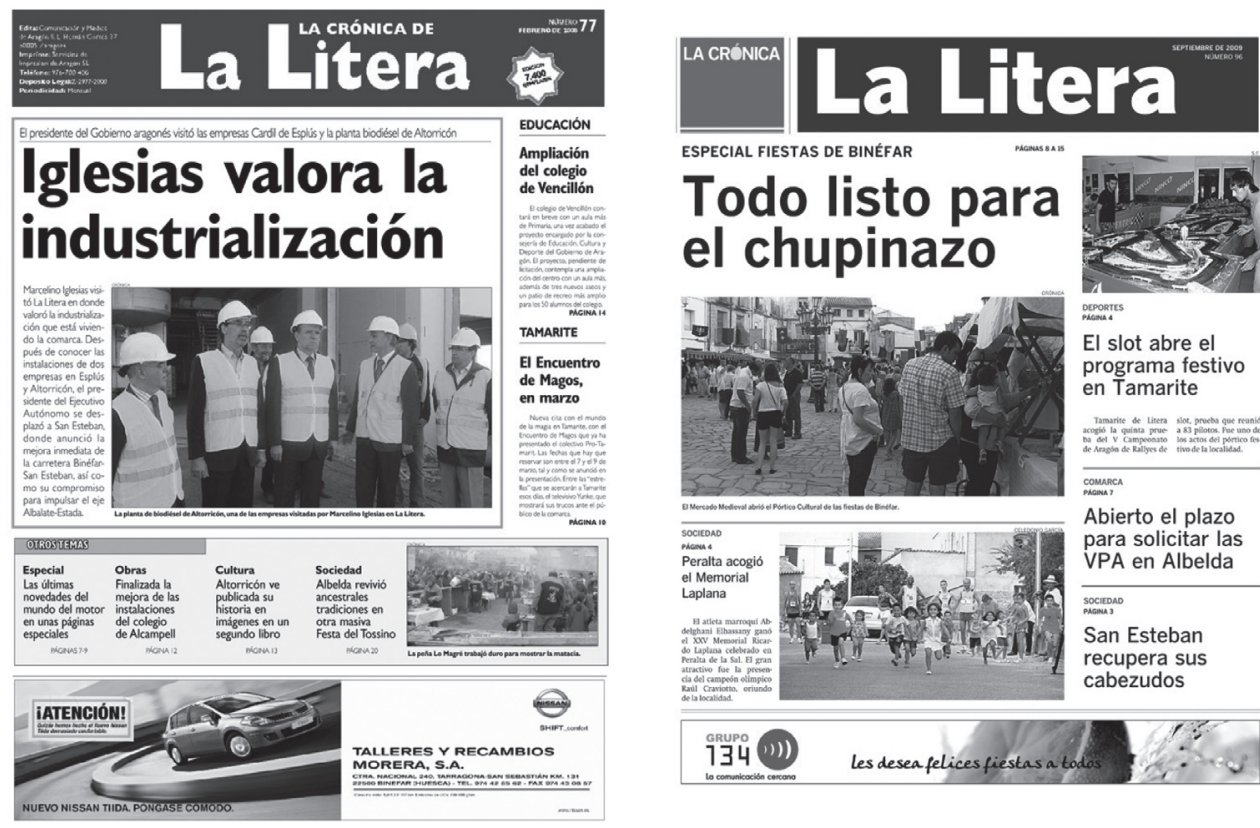

\subsection{Audiencia}

Mensualmente se editan unos 375.000 ejemplares entre las diferentes cabeceras de "La Crónica". Más del $90 \%$ de los mismos se distribuye a través de buzones, tanto en el medio rural como en el urbano. Aunque no existen datos fiables de la audiencia de este soporte, a la hora de establecer las tiradas se divide la población por 2,5.

Uno de los aspectos más destacados de los lectores de "La Crónica" es la identificación del producto entre dos colectivos, la mujer y los jóvenes. Este hecho se constata porque son los dos segmentos que más colaboraciones envían a cada una de "Las Crónicas".

\subsection{Publicidad}

"La Crónica" cumple en muchos lugares un papel estratégico como dinamizador de las economías locales y comarcales, tanto por ser un soporte publicitario como también por las actividades paralelas que organiza, como es el caso del Foro de la Nueva Economía que se desarrolló a mediados 2009 e impulsado desde 134 Comunicación para la provincia de Huesca.

También es un canal que permite la renovación del tejido empresarial dado que facilita a las nuevas iniciativas posicionarse con rapidez en el mercado más 

gratuita en Aragón

próximo en el que muchas veces no hay demasiadas opciones desde el punto de vista comunicativo.

Pero hay que destacar en este aspecto el compromiso institucional que permite que puedan consolidarse "Las Crónicas", y que apuntala este proyecto junto con la colaboración publicitaria de empresas privadas.

El porcentaje de participación de uno y otro varía en cada uno de los territorios, aunque la media de esta presencia es: institucional $40 \%$ y privada $60 \%$. Esta estimación se ha incrementado en los últimos tiempos hacia el lado público debido a la bajada de ingresos que se constata provenientes del ámbito privado.

\section{CONCLUSIONES}

La implantación de las diferentes cabeceras de "La Crónica" se ha realizado aprovechando muy bien un hueco en el mercado informativo entre la población aragonesa que no era bien cubierto por los medios de comunicación existentes.

La preocupación por los contenidos próximos ha sido la clave para que "La Crónica" tenga un respaldo de los lectores que se sienten identificados con el producto al poder leer y ver a través de las imágenes aquellos contenidos informativos que suceden en su entorno, que hacen referencia a las actividades en la que los propios lectores pueden ser protagonistas.

Esa carencia de la que hemos hablado de comunicación de cercanía en Aragón ha permitido que "La Crónica" pudiera asentarse de forma muy rápida en el mercado mediático de la Comunidad Autónoma.

El hecho de ser una publicación gratuita ha sido fundamental para el éxito de esta cabecera que es verdad que ha cuajado más en el ámbito rural que en el urbano y de hecho esto se constata, entre otras cosas, porque ni en Huesca ni en Teruel capital, ni en sus respectivas comarcas, hay una cabecera de "La Crónica".

Un caso aparte es la ciudad de Zaragoza, en la que "La Crónica" de barrio sí se está implantando, apostando también por contenidos que en muchos casos no son los habituales del resto de medios de comunicación que hay en la capital.

El futuro de estas publicaciones pasa por dar un verdadero protagonismo a la estructura social de cada uno de los territorios. Este aspecto debe profundizarse todavía más en los próximos años, ya que en algunas de estas cabeceras existe aún una presencia masiva de contenidos generados por los gabinetes de comunicación institucionales, tanto del Gobierno de Aragón como de la Diputación Provincial, de la Comarca y de los ayuntamientos de la demarcación en la que se distribuye.

De todos modos, "La Crónica" se ha posicionado como un fuerte nexo social y como una publicación que ha incorporado al mundo de la comunicación a mujeres 
y jóvenes. Ese concepto es el que se perfila como una herramienta importante de futuro, ampliando mercados y orientaciones.

"La Crónica" cumple todas las características de prensa periódica gratuita de acuerdo con los descriptores de Santos Díez (2007: 29):

Todos estos periódicos coinciden en los siguientes aspectos: 1) abordan la información local en temas como política, sociedad, ocio, cultura y deporte. 2) Su publicidad es de establecimientos principalmente del sector servicios y pequeño comercio de sus zonas de cobertura. 3) Tienen una periodicidad mensual o semanal. 4) Se distribuyen mediante buzoneo y depósitos. 5) Son una forma de competencia para los medios convencionales. 6) En cuanto a sus contenidos se centran en la actualidad local, deporte, agenda, entrevistas y entretenimiento, junto con publicidad que incide en los sectores relacionados con gremios, hostelería, motor, inmobiliaria e institucional.

Así, "La Crónica" tendrá que definir algo más el modelo de negocio en muchas cabeceras que dependen de forma excesiva de la publicidad institucional. No obstante, hay que entender que la propia estructura del Aragón rural hace inviable muchos proyectos de comunicación si no es con el respaldo de los gobiernos locales, comarcales, provinciales y autonómicos. Como señala Nieto (1984: 99), "que la publicidad sea el único ingreso de las publicaciones gratuitas, plantea situaciones no lejanas a la paradoja. Parece opinión común, que la independencia de una publicación gratuita guarda relación con el volumen de anunciantes: a mayor número de anunciantes corresponde menor posibilidad de intervención de éstos para influir en los contenidos informativos [...]".

Asimismo, el próximo reto también de este producto será su presencia en Internet. En la actualidad, se pueden consultar "Las Crónicas" a través del portal Red Aragón (http://www.redaragon.com/cronicas/), del Grupo Z, pero es verdad que existen carencias como la falta de alguna cabecera y la poca actualización en algunas de ellas.

\section{REFERENCIAS BIBLIOGRÁFICAS}

ESCOLAR, A.: "Leer o no leer, ésa es la cuestión", Cuadernos de Periodistas, no 1 (2004), pp. 39-41.

HERNÁNDEZ NAVARRO, L: La crisis de la prensa escrita. La Jornada. [en línea] 3 de marzo de 2009. [Consulta 6 octubre 2009]. http://www.jornada.unam.mx/2009/03/03/index. php?section=opinion\&article $=019 \mathrm{a} 1 \mathrm{pol}$

LÓPEZ, X.: "El modelo local de prensa gallega redefine estrategias para competir en la nueva era", [en línea] ZER, no 9 (2000). [Consulta 30 septiembre 2009].

NIETO, A. (1984): La prensa gratuita. Pamplona: Eunsa.

SABÉS F. (2002): La radio y la televisión local en Aragón. Lleida: Milenio.

- (2005): Los medios de comunicación y los jóvenes en Aragón. Lleida: Milenio.

- (2006): "Crece la prensa gratuita", Chasqui, no 95, pp. 52-55. 

gratuita en Aragón

- "La prensa gratuita en España: análisis morfológico comparativo de las publicaciones 20 Minutos, Metro, Que! y ADN", Doxa Comunicación, no 7 (2008), pp. 83-108.

- El retroceso de la prensa de calidad entre los jóvenes y su sustitución por los diarios gratuitos. En FERNANDEZ SANZ, J.J.; SANZ ESTABLÉS, C. y RUBIO MORAGA, A.L. (coord.) (2006): Prensa y Periodismo Especializado 3. Guadalajara, pp. 167-184.

SANTOS DÍEZ, T. (2007): El auge de la prensa gratuita en España. Bilbao: Servicio Editorlal de la Universidad del País Vasco.

SIXTO, J.; y BELLÓN, A.: "A prensa gratuita en Galicia a través de tres iniciativas autóctonas: De luns a vernes, Santiago Siete e Tierras de Santiago", Revista F@ro, no 9 (2009).

VINYALS, M.: "Radiografía de la prensa gratuita", Anàlisi, no 35 (2007), pp. 239-244.

\section{Breve semblanza biográfica del autor}

Fernando Sabés Turmo es profesor del Departamento de Periodismo y Ciencias de la Comunicación de la Universidad Autónoma de Barcelona. Entre su bibliografía hay que destacar sus dos últimos libros escritos junto a José Juan Verón Lassa: La eficacia de lo sencillo. Introducción a la práctica del periodismo (2007) y La gestión de la información en la Administración local (2008), ambos publicados en Comunicación Social Ediciones y Publicaciones.

(Recibido el 13-10-2009, aceptado el 12-03-2010) 